\title{
BMJ Open Rapid Assay for Sick Children with Acute Lung infection Study (RASCALS): diagnostic cohort study protocol
}

\begin{abstract}
John Alexander Clark (D) ,, ${ }^{1,2}$ lain Robert Louis Kean, ${ }^{1}$ Martin D Curran, ${ }^{3}$ Fahad Khokhar, ${ }^{4}$ Deborah White, ${ }^{2}$ Esther Daubney, ${ }^{2}$ Andrew Conway Morris, ${ }^{2}$ Vilas Navapurkar, ${ }^{2}$ Josefin Bartholdson Scott, ${ }^{4}$ Mailis Maes, ${ }^{4}$ Rachel Bousfield, ${ }^{2}$ Theodore Gouliouris, ${ }^{5}$ Shruti Agrawal, ${ }^{1,2}$ David Inwald, ${ }^{2}$ Zhenguang Zhang, ${ }^{1}$ M Estée Török (1) , ${ }^{5}$ Stephen Baker, ${ }^{4}$ Nazima Pathan ${ }^{1,2}$
\end{abstract}

To cite: Clark JA, Kean IRL, Curran MD, et al. Rapid Assay for Sick Children with Acute Lung infection Study (RASCALS): diagnostic cohort study protocol. BMJ Open 2021;11:e056197. doi:10.1136/ bmjopen-2021-056197

- Prepublication history and additional supplemental material for this paper are available online. To view these files, please visit the journal online (http://dx.doi.org/10.1136/ bmjopen-2021-056197).

Received 10 August 2021 Accepted 11 November 2021

\section{Check for updates}

(C) Author(s) (or their employer(s)) 2021. Re-use permitted under CC BY. Published by BMJ.

${ }^{1}$ Department of Paediatrics, University of Cambridge, Cambridge, UK

${ }^{2}$ Cambridge University Hospitals NHS Foundation Trust, Cambridge, UK

${ }^{3}$ Clinical Microbiology and Public Health Laboratory, Cambridge, UK

${ }^{4}$ Cambridge Institute of Therapeutic Immunology and Infectious Disease, University of Cambridge, Cambridge, UK

${ }^{5}$ Department of Medicine, University of Cambridge, Cambridge, UK

Correspondence to Dr John Alexander Clark; jac302@cam.ac.uk

\section{ABSTRACT}

Introduction Lower respiratory tract infection (LRTI) is the most commonly treated infection in critically ill children. Pathogens are infrequently identified on routine respiratory culture, and this is a time-consuming process. A syndromic approach to rapid molecular testing that includes a wide range of bacterial and fungal targets has the potential to aid clinical decision making and reduce unnecessary broad spectrum antimicrobial prescribing. Here, we describe a single-centre prospective cohort study investigating the use of a 52-pathogen TaqMan array card (TAC) for LRTI in the paediatric intensive care unit (PICU).

Methods and analysis Critically ill children with suspected LRTI will be enrolled to this 100 patient singlecentre prospective observational study in a PICU in the East of England. Samples will be obtained via routine non-bronchoscopic bronchoalveolar lavage which will be sent for standard microbiology culture in addition to TAC. A blood draw will be obtained via any existing vascular access device. The primary outcomes of the study will be (1) concordance of TAC result with routine culture and 16S rRNA gene sequencing and (2) time of diagnostic result from TAC versus routine culture. Secondary outcomes will include impact of the test on total antimicrobial prescriptions, a description of the inflammatory profile of the lung and blood in response to pneumonia and a description of the clinical experience of medical and nursing staff using TAC.

Ethics and dissemination This study has been approved by the Yorkshire and the Humber-Bradford Leeds Research Ethics Committee (REC reference 20/YH/0089). Informed consent will be obtained from all participants. Results will be published in peer-reviewed publications and international conferences.

Trial registration number NCT04233268.

\section{INTRODUCTION}

Lower respiratory tract infection (LRTI) is a leading cause of admissions to paediatric intensive care units (PICUs) in the UK, and greatest worldwide cause of mortality in

\section{Strengths and limitations of this study}

- This study describes clinical application of a customisable rapid diagnostic respiratory microarray in critically ill children.

- The sampling technique for this project is nonbronchoscopic bronchoalveolar lavage, which is a reliable sampling method for deep respiratory samples, while previous studies have utilised upper airway samples, or endotracheal tube secretions which may be prone to contamination.

- Following this single-centre study, a wider multicentre evaluation will be required to determine feasibility and cost efficiency of this diagnostic method.

- There is no universally agreed diagnostic criteria for pneumonia in children, therefore, clinical suspicion of pneumonia by the treating team rather than a specific set of diagnostic criteria are being used for enrolment.

young children. ${ }^{12}$ PICU physicians regularly prescribe antimicrobial therapy for LRTI, as it is difficult to ascertain whether children have an underlying primary or secondary bacterial infection and withholding or delaying treatment where indicated poses significant risk. ${ }^{3}$ Clinical prediction scores for pneumonia have low specificity in children, ${ }^{45}$ and infection on standard microbiological tests is confirmed in as little as $22 \%$ of treated LRTI. ${ }^{67}$

Rapid diagnostic tests have the potential to reduce untargeted antimicrobial use. ${ }^{8}$ Most currently available molecular respiratory diagnostic panels include common viruses and a limited number of atypical bacterial pathogens such as Legionella pneumophila and Mycoplasma pneumoniae which are difficult to grow on culture. ${ }^{9}{ }^{10}$ Some, such as the FilmArray Pneumonia Panel (BioFire Diagnostics, Utah, 
USA) and Curetis Unyvero pneumonia cartridge (Holzgerlingen, Germany) offer the advantage of being able to be undertaken as a point of care test with sufficient staffing and training. However, they are constrained to testing manufacturer defined molecular targets. ${ }^{11-13}$

The TaqMan array card (TAC) (Thermo Fisher Scientific, California) is a probe-based quantitative PCR (qPCR) assay undertaken in a 384 well plate format. Advantages of TAC are the ability to customise molecular targetsallowing new targets to be incorporated according to local epidemiology, and the ability to run multiple samples on a single card improving cost efficiency. ${ }^{14}$ This may include up to eight clinical samples per card depending on the number of targets sought per sample.

TAC performs reliably on deep respiratory samples obtained from adults with suspected ventilator associated pneumonia. ${ }^{15}$ In children, studies of TAC as an LRTI diagnostic are limited. On extensive search of medical literature, we identified three key studies of TAC in children with suspected pneumonia. One used nasopharyngeal/ oropharyngeal and sputum in hospitalised children. ${ }^{16}$ The second study obtained endotracheal tube (ETT) aspirate samples in 25 children with suspected hospital acquired pneumonia with a limited TAC inclusive of seven bacteria. ${ }^{17}$ The third study tested expectorated sputum samples with an eight bacterial pathogen TAC. ${ }^{18}$ TAC improved diagnostic yield of samples in all studies, ${ }^{16-18}$ but was not used for diagnostic purposes. Obtaining true deep respiratory samples is invasive and technically difficult in children, requiring advanced airway management. These studies used less invasively collected samples as a proxy for bronchoalveolar sampling of the lower respiratory tract. Given the heavy use of systemic antimicrobial therapy in the care of critically ill children, evaluation of TAC to guide antimicrobial therapy requires further evaluation. We are, therefore, undertaking this study to understand the performance and impact of TAC in the PICU setting.

TAC has been evaluated for a number of other paediatric applications, including bloodstream, central nervous system and upper respiratory tract infection, ${ }^{19-24}$ but these studies did not evaluate the utility of TAC in clinical diagnoses. These studies demonstrated high sensitivity and specificity of TAC, but it is difficult to extrapolate this into the clinical setting due to variation in molecular targets selected; regional microbiology; and key differences in clinical practice such as timing of antimicrobial administration.

TAC interpretation requires an approach that is distinct from routinely performed investigations such as culture on which only 1-2 predominant species are generally reported. The TAC array may identify several potential pathogens. Identifying multiple micro-organisms in the lungs may be helpful; however, the interpretation can be challenging for clinicians to determine antimicrobial prescriptions. In the case of hospital-acquired LRTI, this may be due to infiltration of the respiratory tract by bacteria from the dysbiotic intestinal microbiome.
Community-acquired LRTI may also have several pathogens, with co-infection by bacterial and viral pathogens a recognised problem in critically ill children. ${ }^{25}$ The precise identification of multiple organisms in the lungs in parallel should in theory help to guide the use of antimicrobial therapy, but at a clinical level it demands an understanding of how to interpret the data from molecular pathogen assays. To robustly evaluate TAC, comparison of the assay to a culture-independent technique such as metagenomics can identify microorganisms that may have been eliminated due to prior antimicrobial therapy.

While large-scale studies may assess individual molecular targets included on TAC for their performance, a more holistic approach of assessing an entire multipathogen array, including targets at genus and species level, will give an indication of its clinical application.

\section{HYPOTHESIS}

TAC will provide greater sensitivity and a faster turnaround time than standard microbiology tests for the diagnosis of LRTI in critically unwell children.

\section{METHODS AND ANALYSIS \\ Setting}

Patients will be enrolled to the study in a 13 bedded general PICU at Addenbrooke's Hospital, Cambridge, England. The PICU manages neurosurgical and trauma cases but does not support extracorporeal membrane oxygenation or cardiac surgical patients.

\section{Eligibility criteria}

The eligibility criteria are:

1. The child is aged less than 18 years old.

2. The child is receiving mechanical ventilation at the time of enrolment.

3 . The child is commencing or already receiving antimicrobial therapy to treat suspected or confirmed LRTI.

The exclusion criteria are:

1. The patient has a non-survivable illness and is no longer on an active treatment pathway.

2. The child is $<37$ weeks corrected gestation.

These criteria ensure the patient is able to have samples obtained via non-bronchoscopic bronchoalveolar lavage (NB-BAL). Enrolment of children based on antimicrobial prescription for LRTI has been selected rather than the use of clinical features for pneumonia, given there is no consistent and reliable clinical scoring system for this condition. ${ }^{26}{ }^{27}$ Premature infants have been excluded from enrolment due to the sampling procedure not being tested, to our knowledge, in this group of patients, and these infants having distinct pneumonia aetiology that would require separate evaluation.

\section{Selection of participants}

All children admitted to PICU at the study centre will be screened for enrolment into the study by nursing 


$$
\begin{aligned}
& N=\frac{p_{0} q_{0}\left\{z_{1-\alpha / 2}+z_{1-\beta} \sqrt{\frac{p_{1} q_{1}}{p_{0} q_{0}}}\right\}^{2}}{\left(p_{1}-p_{0}\right)^{2}} \\
& q_{0}=1-p_{0} \\
& q_{1}=1-p_{1} \\
& N=\frac{0.22 * 0.78\left\{1.96+0.84 \sqrt{\frac{0.352 * 0.648}{0.22 * 0.78}}\right\}^{2}}{(0.352-0.22)^{2}} \\
& N=85
\end{aligned}
$$

$\mathrm{p}_{0}=$ proportion (incidence) of population $\mathrm{p}_{1}=$ proportion (incidence) of study group $\mathrm{N}=$ sample size for study group $\alpha=$ probability of type I error (usually 0.05 ) $\beta=$ probability of type II error (usually 0.2 ) $\mathrm{Z}=$ critical $\mathrm{Z}$ value for a given $\alpha$ or $\beta$

Figure 1 Power calculation. Calculation generated with ClinCalc. $^{28}$

and medical staff working on the unit. A deferred consent process for up to 48 hours will allow carers to be approached sensitively by the research team, while ensuring samples are obtained within a reasonable time frame to maximise yield. Consent will be obtained via written, electronic and verbal formats via research nursing staff, and permission also obtained for COVID19-related work (online supplemental appendix A and B). Co-enrolment will be permitted in this study so long as there is no impact on the primary outcomes of either project.

\section{Sample size measurement}

This study aims to achieve a relative increase in the sensitivity of lower respiratory tract culture using TAC by $60 \%$. This is a conservative target, given previous paediatric evaluations of TAC have had a relative increase in detection by $>100 \%$ in comparison to routine investigations. ${ }^{16}{ }^{17}$ However, this study is distinct in its sampling approach, patient population and TAC configuration, hence outcomes are difficult to estimate based on available literature.

Previous study on this unit has identified that $22 \%$ of cases of possible LRTI are confirmed on culture. ${ }^{7}$ To increase bacterial confirmation by $60 \%$, a total of 85 patients are required as per power calculation (figure 1). ${ }^{28}$ The total has been rounded up to 100 to account for possible sampling related issues that may occur.

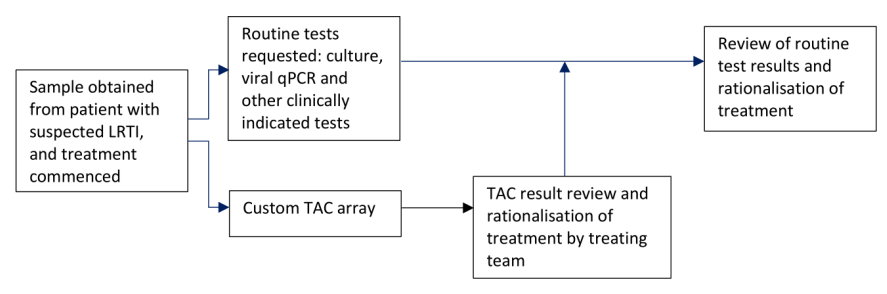

Figure 2 Workflow. LRTI, lower respiratory tract infection; qPCR, quantitative PCR; TAC, Taqman Array Card.

\section{Intervention}

Diagnostic TAC will be undertaken on NB-BAL sample obtained as soon as possible following the time of enrolment (figure 2). The same sample will be used for standard microbiology culture in all patients; however, the clinical team will determine whether viral qPCR panel, extended fungal or mycobacterial cultures or investigations are also indicated. Samples will only be processed during business hours Monday-Friday due to availability of research and laboratory staff.

Samples will be obtained as part of standard practice by physiotherapy, nursing and medical staff that routinely perform the sampling procedure. The results of the test will be reported alongside routine tests, and impact of these results will be investigated. Positive results will be reported with their corresponding cycle threshold (Ct) values, to assist interpretation where a detection may either be a true pathogen or pathobiont. Standard practice in this PICU is for all patients to be reviewed in twice weekly microbiology rounds. In these meetings, the patient's clinical presentation, progress, biochemical and microbiology results, and treatment is reviewed. The clinical team have a low threshold in seeking input from the microbiology team, which provide a 24 hour on-call service. This multidisciplinary approach will assist the clinical team in situations where there are detections on TAC of unclear significance.

\section{Study objectives}

Primary

1. Determine the sensitivity and specificity of TAC in the detection of lower respiratory bacterial pathogens.

2. Compare time to diagnosis of TAC vs standard microbiology culture in the diagnosis of LRTI.

\section{Secondary}

1. Describe the micro-organisms detected on TAC that were not detected using standard diagnostic tests.

2. Describe the lung microbiome of critically ill children with LRTI using $16 \mathrm{~S}$ rRNA gene sequencing.

3. Understand the impact of TAC on total antimicrobial prescriptions in PICU by total prescriptions and by antimicrobial class.

4. Describe the impact of TAC on antimicrobial decision making according to PICU consultants.

5. Describe the host inflammatory response to LRTI in the lung and blood according to pathogen present. 
6. Describe the experience of a tertiary PICU in the implementation of TAC in the clinical setting.

\section{Outcome measures}

Primary

1. TAC result (Ct value).

2. Respiratory culture result (colony forming units $/ \mathrm{mL}$ ).

3. Respiratory viral panel result (Ct value).

4. Time to result (hours).

\section{Secondary}

1. TAC result (Ct value).

2. Blood culture result.

3. Total days of antimicrobial prescriptions in PICU-\% total days of PICU admission and days free of antimicrobial therapy in PICU at 28 days.

4. Reported n (\%) of change in antimicrobial prescription type and duration, and $\mathrm{n}(\%)$ of children able to move out of side rooms due to exclusion of infectious pathogens.

5. Days free of mechanical ventilation at 28 days following PICU admission.

6. Days free of PICU at 28 days following PICU admission.

7. 16S rRNA gene sequencing result-n (\%) of total reads, Shannon's diversity index

8. Cytokine concentration $(\mathrm{pg} / \mathrm{mL})$ in NB-BAL samples and blood.

9. Semistructured interview thematic analysis-description of implementation and interpretation considerations of TAC in the PICU.

\section{SAMPLING AND LABORATORY PROCEDURES NB-BAL sampling}

Deep respiratory samples will be obtained at the time of enrolment via NB-BAL, with some modifications made to the original procedure given SARS-CoV-2 (online supplemental appendix $\mathrm{C}$ and $\mathrm{D}$ ). Saline lavage volume instilled will be $1 \mathrm{~mL} / \mathrm{kg}$ of patient weight to a maximum of 10 $\mathrm{mL}$. Saline is delivered via the in-line suction catheter system and the sample collected in a universal container via a sputum trap. This approach minimises risk of aerosolisation of pathogens such as SARS-CoV-2. Samples will be immediately delivered to the microbiology containment level 3 laboratory and stored at $4^{\circ} \mathrm{C}$ until staff are available to process the samples, between 8:00 and 17:00 hours weekdays. Samples will be split into aliquots for microbiology culture, nucleic acid extraction and any additional clinical tests required.

\section{Nucleic acid extraction}

Up to $750 \mu \mathrm{L}$ of sample will be added to a $2 \mathrm{~mL}$ microtube containing a mixture of ceramic beads with $750 \mu \mathrm{L}$ of $\mathrm{L} 6$ buffer (Qiagen). A minimum of $100 \mathrm{uL}$ will be required for extraction and brought up to $750 \mu \mathrm{L}$ with nuclease free water if low volume. The sample will then be vortexed and incubated for $10 \mathrm{~min}$. Samples will then be processed using an EZ1 virus mini kit (V.2.0) using an EZ1 advanced
XL (Qiagen) in up to 14 samples per run including an L6 buffer-RNase free water negative control. ${ }^{29}$

\section{TaqMan array card}

A custom screening panel was developed and validated via an adult intensive care study that took place in this institution. ${ }^{30}$ Retrospective review of organisms identified on routine tests for severe LRTI in this PICU demonstrated good coverage by this panel. ${ }^{7}$ The targets of the panel are as shown in table 1. Many pathogens are assigned two targets to reduce false positive test interpretations. A target for the MecA gene has been included as it is commonly present in methicillin-resistant Staphylococcus aureus. The TAC includes endogenous control RNase P, internal control MS2 and 18S rRNA gene. TAC configuration was altered on 5 February 2021, to include SARSCoV-2 targets, and opportunistically incorporate Leptospira as this organism was of relevance to the adult ICU service also using the cards. Using the same card for the adult and paediatric service allows samples to be processed in batches of up to four, reducing waste of empty lanes in the card, and minimises laboratory handling time.

For each sample, $50 \mu \mathrm{L}$ of total nucleic acid is added to $50 \mu \mathrm{L}$ of TaqMan Fast Virus 1-step mastermix (Thermo Fisher) and $100 \mu \mathrm{L}$ of RNase free water. A total of $98 \mu \mathrm{L}$ is then added to two lanes of the array. Each lane comprises 48 molecular targets, with the array configured to 96 targets of interest. Therefore, four patient samples are loaded into two lanes each for a total of eight lanes per plate.

The RT-PCR will be undertaken on a QuantStudio 7 Flex (Thermo Fisher) according to the following validated protocol: $50^{\circ} \mathrm{C}$ for $5 \mathrm{~min}, 95^{\circ} \mathrm{C}$ for $20 \mathrm{~s}, 45$ cycles of $95^{\circ} \mathrm{C}$ for $1 \mathrm{~s}, 60^{\circ} \mathrm{C}$ for $20 \mathrm{~s} .{ }^{15}$ qPCR Ct values with clear amplification curves will be reported and documented on the electronic medical record.

\section{Conventional pathogen testing}

Samples will be processed according to Public Health England laboratory standards. For patients that are not immunocompromised, standard media will be used. The sample will be inoculated on chocolate agar and incubated at $35^{\circ} \mathrm{C}-37^{\circ} \mathrm{C}$ supplemented with $5 \%-10 \% \mathrm{CO}_{2}{ }^{31}$ If the patient is immunocompromised, supplementary media will be used, and MacConkey agar and Mannitol salt/Chromogenic agar will be used with the sample incubated in air. ${ }^{31}$ Significant growth constitutes $>10^{4} \mathrm{cfu} / \mathrm{mL}$ on NB-BAL samples, or $>10^{5} \mathrm{cfu} / \mathrm{mL}$ on ETT aspirate. Bacterial organisms will be identified to species or genus level using Matrix-Assisted Laser Desorption/Ionisation Time of Flight (MALDI-TOF) mass spectrometry (Bruker Daltonics, Coventry, UK). Antimicrobial susceptibility testing will be performed using disc diffusion following European Committee on Antimicrobial Susceptibility Testing (EUCAST) guidelines. ${ }^{32}$

An in-house multiplex reverse transcription (RT)-PCR assay will be used for testing NPA and NB-BAL samples for common respiratory viruses: adenovirus, enterovirus, 
Table 1 Molecular targets of the TaqMan diagnostic card for lower respiratory tract infection

\begin{tabular}{lll}
\hline Type & No of targets \\
\hline Bacterial & 16S rRNA gene \\
& Acinetobacter baumannii & 2 \\
\hline Bacteroides fragilis & 3 \\
\hline Bordetella pertussis & 1 \\
\hline Chlamydia pneumoniae & 1 \\
\hline Chlamydia psittaci & 1 \\
\hline Coxiella burnetti & 1 \\
\hline Elizabethkingia meningoseptica & 2 \\
\hline Enterobacter cloacae & 2 \\
\hline Enterobacteriaceae & 1 \\
\hline Enterobacteriaceae Proteus & 1 \\
\hline Enterococcus faecalis* & 1 \\
\hline Enterococcus faecium & * & 2 \\
\hline Escherichia coli & 2 \\
\hline Haemophilus influenzae & 2 \\
\hline Klebsiella pneumoniae & 2 \\
\hline Legionella pneumophilia & 1 \\
\hline Legionella spp & 1 \\
\hline Moraxella catarrhalis & 1 \\
\hline Morganella morganii & 1 \\
\hline Mycobacterium spp & 1 \\
\hline Humanan coronavirus NL63 & 1 \\
\hline Hycoplasma pneumoniae & 2 \\
\hline Mycobacterium tuberculosis & 2 \\
\hline Neisseria meningitidis & 1 \\
\hline Pseudomonas aeruginosa & 2 \\
\hline Serratia marcescens & 2 \\
\hline Staphylococcus aureus & 2 \\
\hline Staphylococcus-coagulase & 1 \\
\hline negative & 1 \\
\hline Staphylococcus epidermidis & 1 \\
\hline Staphylococcus-PVL toxin & 1 \\
\hline Stenotrophomonas maltophilia & 1 \\
\hline Streptococcus pneumoniae & 2 \\
\hline Streptococcus pyogenes & 2 \\
\hline
\end{tabular}

Continued

\begin{tabular}{|c|c|c|}
\hline Type & Target & No of targets \\
\hline & Human coronavirus $229 \mathrm{E}$ & 1 \\
\hline & Human metapneumovirus & 1 \\
\hline & Human parainfluenza virus 1 & 2 \\
\hline & Human parainfluenza virus 2 & 1 \\
\hline & Human parainfluenza virus 3 & 2 \\
\hline & Human parainfluenza virus 4 & 1 \\
\hline & Influenza A & 2 \\
\hline & Influenza A-H1 (2009) & 1 \\
\hline & Influenza A-H3 & 1 \\
\hline & Influenza B & 2 \\
\hline & Parechovirus & 1 \\
\hline & Respiratory syncytial virus (any) & 1 \\
\hline & Respiratory syncytial virus A & 1 \\
\hline & Respiratory syncytial virus B & 1 \\
\hline & Rhinovirus & 2 \\
\hline \multirow[t]{6}{*}{ Fungal } & Aspergillus 28S rRNA gene & 2 \\
\hline & Aspergillus fumigatus & 1 \\
\hline & Candida albicans & 1 \\
\hline & Candida species & 1 \\
\hline & Fungal $18 \mathrm{~S}$ rRNA gene & 1 \\
\hline & Pneumocystis jirovecii & 1 \\
\hline $\begin{array}{l}\text { AMR } \\
\text { gene }\end{array}$ & mecA & 1 \\
\hline \multirow[t]{3}{*}{ Controls } & 18S rRNA gene & 1 \\
\hline & MS2 internal control & 2 \\
\hline & RNase P internal control & 1 \\
\hline $\begin{array}{l}\text { Total } \\
\text { wells }\end{array}$ & & 96 \\
\hline
\end{tabular}

*In enrolments occurring from 5 February 2021 onwards, these targets were replaced to include three targets for SARS-CoV-2, 1 target for Leptospira and an additional target for legionella species.

human metapneumovirus, influenza A and B viruses, parainfluenza virus, rhinovirus and respiratory syncytial virus. This assay is only requested when determined to be of relevance by the clinical team.

If clinically suspected, presence of Aspergillus spp will be tested by culture on Sabouraud dextrose agar with chloramphenicol. Serum will also be tested for the presence of galactomannan antigen. If clinically suspected PCR assay will be used to detect presence of Pneumocystis jirovecii.

\section{S rRNA gene sequencing}

Nucleic acid samples will be quantified using a Qubit 4 fluorometer and Qubit dsDNA HS assay kit (Q32854) (ThermoFisher Scientific, Waltham, Massachusetts, USA). DNA fragment size and quality will be assessed using an Agilent TapeStation 4200 (Agilent, Santa Clara, California, USA). 
After quantification, a sequencing library will be prepared using a 16S rRNA gene Barcoding Kit (SQK16S024), then loaded onto a FLO-MIN106D R9.4.1 flow cell. Sequencing will be undertaken using a MinION nanopore sequencing device (Oxford Nanopore Technologies, UK). Base calling will be undertaken using the EPI2ME platform and data will be stored as FASTQ files. Demultiplexing will be completed with guppy_barcoder V.5.0.11, adapters will be trimmed with porechop 0.2.4, and reads filtered with NanoFilt V.2.8.0. Filtered reads will be classified using Kraken2. ${ }^{33}$ Results will be for research purposes only and not distributed to the clinical team.

\section{Blood sampling}

Blood will be obtained for research if the child is undergoing venepuncture for clinical purposes or if there is an existing vascular access device. The maximum volume obtained will be $1 \mathrm{~mL} / \mathrm{kg}$ to a maximum $10 \mathrm{~mL}$ in keeping with WHO guidelines. ${ }^{34}$ Collected blood will be stored in a $4^{\circ} \mathrm{C}$ refrigerator on the ward until the research team are able to process samples. EDTA tubes will be spun at 1300 $\mathrm{g}$ for $10 \mathrm{~min}$ at $4^{\circ} \mathrm{C}$, and aliquots stored at $-20^{\circ} \mathrm{C}$ until batch processing.

\section{Cytokine assay}

Cytokines will be quantified from NB-BAL and plasma samples using a Bio-Plex Pro Human Cytokine Screening 48-plex kit (Bio-Rad) using standard methods. ${ }^{35}$ This assay will be undertaken on aliquots of NB-BAL stored at $-80^{\circ} \mathrm{C}$ until batch processing. NB-BAL will be undiluted while plasma will be diluted with standard diluent $\mathrm{HB}$ as per manufacturer recommendations dependent on limits of detection according to the generated standard curves.

\section{Survey and semistructured interviews}

Senior medical staff on the PICU will be surveyed regarding their antimicrobial decision making after TAC result becomes available (online supplemental appendix E). Survey invitation will be sent via Research Eletronic Data Capture (REDCap) to the hospital email address of the duty PICU consultant when the TAC result becomes available.

Semistructured interviews will be undertaken with PICU nursing and medical staff at the end of the study. They will be advertised using posters and via the internal newsletter to staff. Voluntary consent will be obtained (online supplemental appendix F and G). Sessions will be facilitated by a member of the research team and recorded. Themes and descriptions will be analysed using NVivo V.11. ${ }^{36}$ These interviews will capture the experience of staff in implementing TAC into clinical practice, the benefits and downsides to the test, and interpretation of the test.

\section{Patient and public involvement}

Carers of children admitted to PICU identified rapid molecular diagnostic tests for early rationalisation of antimicrobial therapy to be a high priority area for research. They ranked this fourth of 73 potential national priority areas for PICU research in a 2019 multicentre survey. ${ }^{37}$ As this study is investigating acute clinical decision making of critical care clinicians, patients were not involved in the design of scientific study methodology. The research team will be engaging interested families who have had children admitted to this PICU for their input in the dissemination of research findings and for additional feedback on the design of patient information and consent materials.

\section{Study status}

Enrolment commenced on 10 April 2020 and enrolment is expected to be completed prior to March 2023.

\section{DATA MANAGEMENT \\ Database}

Data will be obtained from the electronic medical record and recorded on REDCap, a secure data management system which will be hosted by the University of Cambridge ${ }^{38}$ Data collected will include demographics, physiological parameters, paediatric index of mortality 3 score ${ }^{39}$ treatment received and investigation results from the time of presentation to hospital for the acute illness to discharge.

\section{Statistical analysis plan}

Data will be analysed in $\mathrm{R}^{40}$ Demographic data will be reported using simple descriptive statistics including mean and standard deviation (SD). For comparisons of demographic data between groups, skewed data using non-parametric tests including Mann-Whitney $U$ test and Kruskal-Wallis test. Normally distributed data will be compared with Student's t-tests. Group correlations of quantitative data will be tested with Spearman correlation test.

All TAC detections from NB-BAL samples will be reported using the mean and range of $\mathrm{Ct}$ values for each molecular target. This will be compared with growth on culture in $\mathrm{cfu} / \mathrm{mL}$ or $\mathrm{Ct}$ value for routine $\mathrm{qPCR}$ testing. A positive TAC result will be considered a Ct of 32 or below, which was found to correlate with microbiology culture thresholds in a previous investigation. ${ }^{30}$

Time to reportable results will be recorded as the time taken from collection time of the sample, which will be entered by nursing staff into the electronic medical record. The comparison of time to result of TAC versus culture will be assessed with Wilcoxon signed ranks test.

Cytokine results will be analysed using a supportvector machine approach to explore profiles of different infections.

\section{ETHICS AND DISSEMINATION}

\section{Ethics and registration}

The study is jointly sponsored by Cambridge University Hospitals NHS Foundation Trust and the University of Cambridge. The study was approved by the Yorkshire and 
the Humber-Bradford Leeds Research Ethics Committee on 26 March 2020 (REC reference 20/YH/0089). The amended, current version of the protocol (8.0) was approved by the REC on 2 July 2021. Amendments to this study have primarily been a result of the SARSCoV-2 pandemic. Additional work packages investigating SARS-CoV-2 infection in children under the overarching RASCALS project are subject to separate protocol and analysis that are not outlined in this paper.

Protocol amendments will be communicated to participants when changes are made that result in a change in procedures for the individual. Participants will be pseudonymised via creation of a sequential study ID.

\section{INFORMED CONSENT PROCEDURES}

Consent will be obtained in written, electronic or verbal format as approved by the ethics committee. While written consent is preferred, alternative consent procedures were introduced due to the COVID-19 pandemic. Deferred consent for up to 48 hours will be permitted to allow time critical samples to be obtained, while also ensuring families are approached sensitively in the PICU.

\section{DISSEMINATION}

Findings from this project will be reported in peerreviewed journals and international conferences.

Twitter John Alexander Clark @doctorjclark, Fahad Khokhar @effkay88, Deborah White @DebbieWhite3, Andrew Conway Morris @andymoz78, Josefin Bartholdson Scott @jbartholdson, Mailis Maes @MaesMailis, Rachel Bousfield @Dr_Rach_Bo, Shruti Agrawal @shagrawal, David Inwald @Dlawni, M Estée Török @EsteeTorok, Stephen Baker @baker_lab_cam and Nazima Pathan @drnazimapathan

Acknowledgements The authors would like to thank the staff at Addenbrooke's Hospital for supporting the development and implementation of this study; Helen Starace and Colin Hamilton (physiotherapists) for assisting with developing NB-BAL sampling procedure; Adam Palmer (PICU data manager) for providing admissions data; and Claire Jenkins (scientist) for assisting with sample workflow.

Contributors JAC, MDC, ACM, VN, MET, SB and NP conceived the study design; JAC, MDC, ACM, VN, MET, SB and NP designed the study protocol; JAC, IRLK, MDC, FK, JBS, MM, and SB developed laboratory methods for the study. JAC, DW, ED, SA and DI developed sampling procedure protocol; JAC, IRLK, FK, ACM, RB, TG, ZZ, SB and NP developed the statistical analysis plan; JAC, IRLK, MDC, FK, DW, ED, ACM, VN, RB, TG, SA, ZZ, SB and NP drafted this manuscript.

Funding This project is funded by the Addenbrooke's Charitable Trust, Cambridge University Hospitals (900240) (JAC, NP, MET and SB) which will provide funding for consumables, $16 \mathrm{~S}$ rRNA gene sequencing of samples and inflammatory profiling; in addition to the NIHR Cambridge Biomedical Research Centre. The authors also receive support from the Gates Cambridge Trust (OPP1144) (JAC); the Academy of Medical Sciences (MET); Wellcome Trust [215515] (SB); Wellcome Trust Clinical Research Career Development Fellowship (WT 2055214/Z/16/Z) (ACM) MRC Clinician Scientist Fellowship [MR/V006118/1] (ACM); and Action Medical Research (NP, SB, MET) (GN2751, GN2903). This work was supported, in whole or in part, by the Bill \& Melinda Gates Foundation (OPP1144). Under the grant conditions of the Foundation, a Creative Commons Attribution 4.0 Generic License has already been assigned to the Author Accepted Manuscript version that might arise from this submission.

Competing interests MDC is the inventor on a patent held by the Secretary of State for Health (UK government) EP2788503, which covers some of the genetic sequences used in this study. VN is a founder, director and shareholder in Cambridge Infection Diagnostics (CID) which is a commercial company aimed at developing molecular diagnostics in infection and antimicrobial and AMR stewardship. ACM, SB and ED are members of the Scientific Advisory Board of
Cambridge Infection Diagnostics (CID). TG has received a research grant from Shionogi. All other authors declare no conflict of interest.

Patient consent for publication Not applicable.

Provenance and peer review Not commissioned; externally peer reviewed.

Supplemental material This content has been supplied by the author(s). It has not been vetted by BMJ Publishing Group Limited (BMJ) and may not have been peer-reviewed. Any opinions or recommendations discussed are solely those of the author(s) and are not endorsed by BMJ. BMJ disclaims all liability and responsibility arising from any reliance placed on the content. Where the content includes any translated material, BMJ does not warrant the accuracy and reliability of the translations (including but not limited to local regulations, clinical guidelines, terminology, drug names and drug dosages), and is not responsible for any error and/or omissions arising from translation and adaptation or otherwise.

Open access This is an open access article distributed in accordance with the Creative Commons Attribution 4.0 Unported (CC BY 4.0) license, which permits others to copy, redistribute, remix, transform and build upon this work for any purpose, provided the original work is properly cited, a link to the licence is given, and indication of whether changes were made. See: https://creativecommons.org/ licenses/by/4.0/.

ORCID iDs

John Alexander Clark http://orcid.org/0000-0001-6916-9195

M Estée Török http://orcid.org/0000-0001-9098-8590

\section{REFERENCES}

1 GBD 2016 Causes of Death Collaborators. Global, regional, and national age-sex specific mortality for 264 causes of death, 19802016: a systematic analysis for the global burden of disease study 2016. Lancet 2017;390:1151-210.

2 Paediatric Intensive Care Audit Network. Paediatric Intensive Care Audit Network Annual Report 2019; 2019.

3 Kumar A, Roberts D, Wood KE, et al. Duration of hypotension before initiation of effective antimicrobial therapy is the critical determinant of survival in human septic shock. Crit Care Med 2006;34:1589-96.

4 da Silva PSL, de Aguiar VE, de Carvalho WB, et al. Value of clinical pulmonary infection score in critically ill children as a surrogate for diagnosis of ventilator-associated pneumonia. J Crit Care 2014;29:545-50.

5 Sachdev A, Chugh K, Sethi M, et al. Clinical pulmonary infection score to diagnose ventilator-associated pneumonia in children. Indian Pediatr 2011;48:949-54.

6 Stocker M, Ferrao E, Banya W, et al. Antibiotic surveillance on a paediatric intensive care unit: easy attainable strategy at low costs and resources. BMC Pediatr 2012;12:196.

7 Clark J, White D, Daubney E, et al. Low diagnostic yield and time to diagnostic confirmation results in prolonged use of antimicrobials in critically ill children. Wellcome Open Res 2021;6:119.

8 Byington CL, Castillo H, Gerber K, et al. The effect of rapid respiratory viral diagnostic testing on antibiotic use in a children's Hospital. Arch Pediatr Adolesc Med 2002;156:1230-4.

9 Whiley H, Taylor M. Legionella detection by culture and qPCR: comparing apples and oranges. Crit Rev Microbiol 2016;42:65-74.

10 Daxboeck F, Krause R, Wenisch C. Laboratory diagnosis of Mycoplasma pneumoniae infection. Clin Microbiol Infect 2003;9:263-73.

11 Papan C, Meyer-Buehn M, Laniado G, et al. Assessment of the multiplex PCR-based assay Unyvero pneumonia application for detection of bacterial pathogens and antibiotic resistance genes in children and neonates. Infection 2018;46:189-96.

$12 \mathrm{Li} \mathrm{J}$, Tao Y, Tang M, et al. Rapid detection of respiratory organisms with the FilmArray respiratory panel in a large children's hospital in China. BMC Infect Dis 2018;18:510.

13 Leber AL, Everhart K, Daly JA, et al. Multicenter evaluation of BioFire FilmArray respiratory panel 2 for detection of viruses and bacteria in nasopharyngeal swab samples. J Clin Microbiol 2018;56:e01945-17.

14 Kodani M, Yang G, Conklin LM, et al. Application of TaqMan lowdensity arrays for simultaneous detection of multiple respiratory pathogens. J Clin Microbiol 2011;49:2175-82.

15 Jones NK, Conway Morris A, Curran MD, et al. Evaluating the use of a 22-pathogen TaqMan array card for rapid diagnosis of respiratory pathogens in intensive care. J Med Microbiol 2020;69:971-8.

16 Wolff BJ, Bramley AM, Thurman KA, et al. Improved detection of respiratory pathogens by use of high-quality sputum with TaqMan array card technology. J Clin Microbiol 2017;55:110-21. 
17 Mansour MGE, Bendary S. Hospital-acquired pneumonia in critically ill children: incidence, risk factors, outcome and diagnosis with insight on the novel diagnostic technique of multiplex polymerase chain reaction. Egypt J Med Hum Genet 2012;13:99-105.

$18 \mathrm{Hu}$ L, Han B, Tong Q, et al. Detection of eight respiratory bacterial pathogens based on multiplex real-time PCR with fluorescence melting curve analysis. Can J Infect Dis Med Microbiol 2020;2020:1-11.

19 Diaz MH, Waller JL, Theodore MJ, et al. Development and implementation of multiplex TaqMan array cards for specimen testing at child health and mortality prevention surveillance site laboratories. Clin Infect Dis 2019;69:S311-21.

20 Zhao C, Wang X, Zhang C, et al. Development of a TaqMan array card to target 21 purulent meningitis-related pathogens. BMC Infect Dis 2019;19:289.

21 Onyango CO, Loparev V, Lidechi S, et al. Evaluation of a TaqMan array card (TAC) for detection of central nervous system infections. J Clin Microbiol 2017;55:2035-44.

22 Zhang $C$, Zheng $X$, Zhao $C$, et al. Detection of pathogenic microorganisms from bloodstream infection specimens using TaqMan array card technology. Sci Rep 2018;8:12828.

23 Liu K, Jing H, Chen $\mathrm{Y}$, et al. Evaluation of TaqMan array card (TAC) for the detection of 28 respiratory pathogens. BMC Infect Dis 2020;20:820.

24 Caserta MT, Yang H, Gill SR, et al. Viral respiratory infections in preterm infants during and after hospitalization. $J$ Pediatr 2017;182:53-8.

25 Diaz MH, Cross KE, Benitez AJ, et al. Identification of bacterial and viral Codetections with Mycoplasma pneumoniae using the TaqMan array card in patients hospitalized with community-acquired pneumonia. Open Forum Infect Dis 2016;3: ofw071.

26 Goodman D, Crocker ME, Pervaiz F, et al. Challenges in the diagnosis of paediatric pneumonia in intervention field trials: recommendations from a pneumonia field trial working group. Lancet Respir Med 2019;7:1068-83.

27 Foglia E, Meier MD, Elward A. Ventilator-Associated pneumonia in neonatal and pediatric intensive care unit patients. Clin Microbiol Rev 2007;20:409-25.

28 Kane S. Sample size calculator, 2021. ClinCalc

29 Qiagen. EZ1 Virus Handbook, 2010.

30 Navapurkar Vet al. Development and implementation of a customised rapid syndromic diagnostic test for severe pneumonia. medRxiv 2020:2020.06.02.20118489.

31 Public Health England. UK Standards for Microbiology Investigations: Investigation of bronchoalveolar lavage, sputum and associated specimens, 2019

32 EUCAST. EUCAST disk diffusion method, 2021

33 Wood DE, Lu J, Langmead B. Improved metagenomic analysis with Kraken 2. Genome Biol 2019;20:257.

34 Howie SRC. Blood sample volumes in child health research: review of safe limits. Bull World Health Organ 2011;89:46-53.

35 Bio-Rad. Bio-Plex Pro Human Cytokine Assays - Instruction Manual, 2021.

36 QRS International Pty Ltd. Nvivo qualitvative data analysis software, 2018

37 Tume LN, Menzies JC, Ray S, et al. Research priorities for U.K. pediatric critical care in 2019: healthcare professionals' and parents' perspectives. Pediatr Crit Care Med 2021;22:e294-301.

38 Harris PA, Taylor R, Thielke R, et al. Research electronic data capture (REDCap)--a metadata-driven methodology and workflow process for providing translational research informatics support. J Biomed Inform 2009;42:377-81.

39 Straney L, Clements A, Parslow RC, et al. Paediatric index of mortality 3: an updated model for predicting mortality in pediatric intensive care*. Pediatr Crit Care Med 2013;14:673-81.

40 RStudio Team. RStudio: integrated development for R, 2019 